\title{
A DIAGRAM OF THE THERMAL PROPERTIES OF GAS MIXTURE AND A DIAGRAM FOR THE CALCULATION OF NOZZLES.
}


DIAGRaM OF THERMAL PROPERTIES OF GAS MIXTURE, ETC. 659

\section{A DIAGRAM OF THE THERMAL PROPERTIES OF GAS MIXTURE}

AND

A DIAGRAM FOR THE CALCULATION OF NOZZLES.

By W. H. H. GIBSON, B.Sc., B.E.

(Waiter and Eliza Halt Feliow in Engrnegring, Untversity of Sydnet).

\section{Heat Engine Calculations.}

(1) The calculations which are usually necessary in dealing with the working substance of a heat engine may be classified under one or more of the following headings:-

(a) Calculation of the properties of state,

Pressure,

Specific volume,

Temperature,

Entropy,

Total heat,

Internal energy,

when the values of any independent pair of them are known.

(b) Calculation of heat received or rejected;

at constant pressure,

at constant volume,

at constant temperature.

(c) Calculation of work done in expansion or received in compression;

at constant entropy (adiabatic),

at constant temperature (isothermal),

at constant pressure.

(d) Transfer of working substance from one vessel to another and flow through nozzles or orifices. 
The object of Fig. 1, see p. 667,* here described is the simplification of these calculations, as applied to the working substance of the internal combustion engine.

\section{Methods of Simplifying Calculatron.}

(2) For the reduction of tedious detail calculations, with their consequent liability to error, three methods are in general use: Tables, Curves and Scales. The outstanding example of the last-named is the slide-rule, but tables and curves are uced so extensively that it is difficult to name their chief applications.

Tables.-Tables are used principally in calculations requiring greater accuracy than can be obtained with convonienee by graphical methods. If the calculation of the tabulated values is correct and is carried far enough, their accuracy depends only, on that of the data on which they are based. Intermediate values are usually obtained by approximate methods of interpolation, which, however, still give more accurate results than graphical methods in their normal applications. Complete tabulation of quantities which depend on two variables gives a set of tables rather inconvenient to use, and particularly so if there are several derived quantities, of which any two may be the data in the case to which the tables are applied. In general, work from tables is more accurate but considerably slower than from suitable eurves or diagrams.

Curves.-A simple relation between two variables is conveniently, represented by a plane curve. This method may be extended to cover the case of a third variable, dependent on the others, by the use of a three-dimensional model, but such representations, whilo sometimes useful for demonstration, are of little or no value for numerical calculations. It is usual in this case to retain the plane curves and to plot them in sets, each curve representing the relation between two of the variables for a fixed value of the third: Similar sets of curves may be plotted for fixed values of other functions of the same two variables, giving the familiar network of the Mollier and other thermodynamie diagrams. In the use of these, the space between adjacent curres must be subdivided for intermediate values; thus each curve is, in effect, a division on a scale of varying length, formed by its set. The principal diffi-

* These Diagrams may be obtained separately mounted on stiff cards, size 11 by 8 , price $2 s$. each, on application to the Secretary. 
culty in the use of these diagrams occurs when it is necessary to determine the point representing intermediate values from each of two of the sets of curves, especially when the respective curves intersect at an acute angle. When several sets of eurves are drawn, it is also difficult to number them closely without confusion.

Scales.-For the application which has been considered, seales Jave several advantages. In the first place, one scale may be used to replace a complete set of curves, in a three-variable relation. Divisions on a linear scale can be subdivided by eye with greater ease and accuracy than the spaces between curves. Finally, the scales may be isolated so that each can be closely numbered. without any interference with the others.

From these considerations, an Alignment Diagram* (or Nomogram) was chosen as a suitable and convenient method for the representation of the relations between the properties of state.

\section{Table of Symbols and Units.}

(3) The symbols and units used are given in the following: Table:-

Symbol.
$\mathrm{P}$
$\mathrm{V}$
$\mathrm{T}$
$\phi$

$\mathrm{H}$ Total Heat

E Internal Energy

\section{Cuits.}

Lb. per sq. in.

Cu. ft. per lb.

Degrees Fahrenheit Absolute.

"Pounds," or Entropy received in absorbing $N$ B. Th. Us. at $N^{\circ} \mathrm{F}$. Abs., per pound.

British Thermal Units, per pound.

British Thermal Units, per pound.

\section{Specific Heats of Gas Mixture.}

(4) The relations between the properties of state depend on the specific heats of the gas mixture. The specific heats used in the construction of the Diagram are derived from an expression

* The principal works on the alignment diagram, or nomogram, are those of d'Ocagne: “Traité de Nomographie," Paris, 1899 ; "Calcul graphique et Nomograpbie," Paris, 1914. A bibliography of the subject is given in a paper presented to the Institution by F. Leigh Martineau and A. Marshall Arter. See Proc. I.A.E., Vol. XII., p. 187. 
given by Stodola* as an approximation, suitable for the gas mixture of the internal combustion engine, to Pier's values for the specific heat at constant volume of different gases and vapours, and used for the construction of a thermodynamic diagram. By assuming a mean molecular weight of 28 for the gas mixture, Morley† reduced Stodola's expression from kg.-calories per $\mathrm{kg}$.-molecule per degree Centigrade to the customary form of thermal units per unit mass per unit temperature, applicable equally to kilogram-Centigrade, pound-centigrade, or poundFahrenheit units. Also, by assuming a constant difference between the specific heats, he obtained a similar expression for the specific heat at constant pressure. In the same paper, the values thus obtained were compared with others, due to Clerk and to the Gaseous Explosions Committee of the British Association. They have also been used by Alexander in the construction of a temperature-entropy diagram with superposed scales of total heat and internal energy.

In the diagram given by Stodola, the specific heat at constant volume, expressed in kilogram-calories for one kilogram-molecule of substance per degree Centigrade, is

$$
4 \cdot 67+1 \cdot 736 \times 10^{-3} \theta-0 \cdot 203 \times 10^{-6} \theta^{2}
$$

at the temperature of $\theta$ degrees Centigrade absolute.

Morley divides by the molecular weight, taken as 28 , and from the corresponding density fixes the difference between the specific heats as 0.0707 . The specific heats, at constant volume and constant pressure respectively, are then given by

$$
\begin{aligned}
& \mathrm{C}_{v}=0 \cdot 167+0^{\circ} 000062 \theta-0.725 \times 10^{-8} \theta^{2} \\
& \mathrm{C}_{p}=0 \cdot 2377+0^{\circ} 000062 \theta-0.725 \times 10^{-8} \theta^{2} .
\end{aligned}
$$

Expressed in terms of Fahrenheit absolute temperature $\mathrm{T}$, as in the table of units, $\S 3$, these values become

$$
\begin{aligned}
& \mathrm{C}_{\nu}=0 \cdot 167+3 \cdot 44 \times 10^{-5} \mathrm{~T}-2 \cdot 24 \times 10^{-9} \mathrm{~T}^{2} \\
& \mathrm{C}_{p}=0 \cdot 2377+3 \cdot 44 \times 10^{-5} \mathrm{~T}-2 \cdot 24 \times 10^{-9} \mathrm{~T}^{2} .
\end{aligned}
$$

- A. Stodola, "Zum Wirkungsgrad der Explosionsturbine." (Zeitschrift des Vereives deutscher Ingenieure, 22nd June, 1912, pp. 1005-1009.)

† T. B. Morley, "The Limits of Efficiency of Gas Engines." (Engineering, 18th December, 1914, pp. 734-735.)

$\ddagger$ W. Alexander, "An Energy Diagram for Gas Mixture, and some of it Uses." (Proc. I. Mech. E., May, 1917, pp. 333-356.) 


\section{Construction of the Gas Mixture Diagram.}

(5) The relation between pressure, specific volume, and temperature is given by

$$
\begin{aligned}
144 \mathrm{PV} & =778\left(\mathrm{C}_{p}-\mathrm{C}_{\vartheta}\right) \mathrm{T} \\
\mathrm{PV} & =0.382 \mathrm{~T} .
\end{aligned}
$$

or

Expressing this in the form

$$
\log \mathrm{P}+\log \mathrm{V}=\log \mathrm{T}+\log (0 \cdot 382),
$$

we have a simple addition formula, which can be represented on an alignment diagram by three straight parallel scales, divided in proportion to the logarithms of $\mathrm{P}, \mathrm{V}$, and $\mathrm{T}$ respectively. The relative lengths of the scales for a given range will be determined by the arrangement and spacing adopted.

In Fig. 1, the seales representing this relation are those marked $P, V$, and $T_{1}$. The lengths for a range of $10: 1$ are: on the $\mathrm{T}$ scale, 10 in.; on the $\mathrm{P}$ scale, 5 in.; and, on the $\mathrm{V}$ scale, $3 \cdot 33_{3}$ in. The distance between the $P$ and $V$ scales is therefore half that between the $\mathrm{V}$ and $\mathrm{T}$ scales. As pressure varies: inversely as volume at constant temperature, and the $\mathrm{V}$ scale is placed between the $P$ and $T$ scales, the $V$ and $P$ scales are necessarily divided in opposite directions; increasing values of pressure are scaled downward, increasing values of volume upward. Temperature, increasing with volume or pressure, is also scaled upward.

(6) The increment of entropy is defined as the heat received divided by the temperature at which it is received. As the heat received is the sum of the increment of internal energy and the work done, this may be expressed by the equation

or

$$
\begin{aligned}
& d \phi=(d \mathrm{E}+144 . \mathrm{P} . d V / 778) / \mathrm{T} \\
& d \phi=\left(\mathrm{C}_{v} . d \mathrm{~T} \div 144 . \mathrm{P} . d \mathrm{~V} / 778\right) / \mathrm{T} .
\end{aligned}
$$

By applying the value for specific heat at constant volume $(\$ 4)$, and the relation between pressure, volume, and temperature $(\$ 5)$, this may be expressed in two variables and integrated. To fix the constant of integration, it is necessary to assign arbitrary values of these variables, corresponding to zero entropy. The values chosen are: temperature, $492^{\circ} \mathrm{F}$. Abs. $\left(=32^{\circ} \mathrm{F}\right.$.); pressure, $14.7 \mathrm{lb}$. per sq. in. ("atmospheric"); with the corresponding volume of $12.78 \mathrm{cu}$. ft. per $\mathrm{lb}$.

With these conditions, entropy may be expressed in terms of temperature and pressure by the equation

$$
\begin{gathered}
\phi=0.2377 \log _{e} \mathrm{~T}+3.44 \times 10^{-5} \mathrm{~T}-1.12 \times 10-9 \mathrm{~T}^{2} \\
-0.0707 \log _{e} \mathrm{P}-1.300
\end{gathered}
$$


or in terms of temperature and volume, by

$$
\begin{gathered}
\phi=0.167 \log _{e} \mathrm{~T}+344 \times 10^{-5} \mathrm{~T}-1 \cdot 12 \times 10^{-9} \mathrm{~T}^{2} \\
+0.0707 \log _{e} \mathrm{~V}-1.232 .
\end{gathered}
$$

Reducing the logarithms to the base 10 , these equations can be expressed in the form

$$
\begin{gathered}
\phi=0.547_{2}\left(\log _{10} \mathrm{~T}+6.30 \times 10^{-5} \mathrm{~T}-2.04_{4} \times 10^{-9} \mathrm{~T}^{2}\right) \\
-0.1628 \log _{10} \mathrm{P}-1.300 \\
=0.384_{5}\left(\log _{10} \mathrm{~T}+8.96 \times 10^{-5} \mathrm{~T}-2.91 \times 10^{-9} \mathrm{~T}^{2}\right) \\
+0.1628 \log _{10} \mathrm{~V}-1.232 .
\end{gathered}
$$

The scale $\mathrm{T}_{2}$ is divided in proportion to the value of the expression $\left(\log _{10} \mathrm{~T}+6 \cdot 30 \times 105 \mathrm{~T}-2 \cdot 04, \times 10^{-9} \mathrm{~T}^{2}\right)$, unit value of this function being represented by a length of $10 \mathrm{in.}$ on the scale. As unit value of $\log _{10} \mathrm{P}$ is represented on the $\mathrm{P}$ scale by 5 in. $(\$ 5)$, or algebraically by $-\bar{j}$ in., taking the upward direction as positive, entropy, according to the equation in temperature and pressure, is given by a uniform scale, marked $\phi$, so placed between $\mathrm{P}$ and $\mathrm{T}_{2}$ that the distance between $\mathrm{P}$ and $\phi$ is to the distance between $\phi$ and $\mathrm{T}_{2}$ in the ratio of $0.547_{2}$ to $0.1628 \times 2$. At constant pressure, a length of $10 \mathrm{in}$. on the scale $T_{9}$ corresponds to a change in entropy of $0.547_{2}$. The corresponding length on the scale $\phi$ is 10 in. $\times 0.547_{2} / 0.872_{8}$; therefore unit change of entropy is indicated on the scale $\phi$ by a length of $10 \mathrm{in} .0 .872_{8}$ or $11.46 \mathrm{in}$.

By making $10 \mathrm{in}$. on the scale $\mathrm{T}_{3}$ correspond to unit value of the function $\left.\left(\log _{10} \mathrm{~T}+8.96 \times 10^{-5} \mathrm{~T}-2.91 \times 10-4\right) \mathrm{T}^{2}\right)$, and spacing $\mathrm{T}_{3}$ so that the distance between $\phi$ and $\mathrm{T}_{3}$ is to the distance between $\mathrm{V}$ and $\phi$ as $0.1628 \times 3$ is to $0.384_{5}$ (since unit ralue of $\log _{10} \mathrm{~V}$ is given by $3 \cdot 33_{3}$ in. on the scale $V$ ), the same scale $\phi$ may be used to represent entropy, expressed in terms of temperature and volume on the scales $\mathrm{T}_{3}$ and $\mathrm{V}$ respectively, the sum of $(0.1628 \times 3)$ and $0 \cdot 384_{5}$ being $0 \cdot 872_{9}$, and the scale for unit entropy therefore $11 \cdot 46$ in, again.

A check on construetion is provided by the fact that a line crossing two of the $T$ scales at any one temperature must also cross the third at the same temperature. For as one scale for each of the properties pressure, volume, and entropy is used to represent that property in two equations, any line which indicates, for example, the volume corresponding to a given pressure and temperature, and also the entropy corresponding to the same pressure and temperature, by its intersections with two of the groups of scales, must also show the temperature corresponding to the same volume and entropy on the only remaining scale. As two 
independent properties, such as pressure and temperature, fix all the others, this is necessarily the same temperature.

(7) The internal energy is found by integrating the expression for the increment of internal energy

$$
d \mathrm{E}=\mathrm{C}_{\imath} \cdot d \mathrm{~T}
$$

already used in deriving the entropy. The constant of integration is again fixed by taking the zero of internal energy at the temperature of $492^{\circ} \mathrm{F}$. Abs. The total heat is the sum of the internal energy and the external energy, and is given by

$$
\mathrm{H}=\mathrm{E}+144 . \mathrm{P} . \mathrm{V} / 778=\mathrm{E}+0.0707 . \mathrm{T} \text {. }
$$

Substituting the value of $\mathrm{C}_{v}$ and integrating, the total heat and internal energy are given by the equations

$$
\begin{aligned}
& \mathrm{H}=0.2377 \mathrm{~T}+1.72 \times 10^{-5} \mathrm{~T}^{2}-7.46 \times 10^{-10} \mathrm{~T}^{3}-86.25 \\
& \mathrm{E}=0.167 \mathrm{~T}+1.72 \times 10^{-5} \mathrm{~T}^{2}-7.46 \times 10^{-10} \mathrm{~T}^{3}-86.25 .
\end{aligned}
$$

As these are both functions in which $\mathrm{T}$ is the only variable, either of them can be plotted on the opposite side of one of the temperature scales. In Fig. 1, total heat is shown on the scale $H$. against $\mathbf{T}_{1}$, and internal energy on the scale $\mathrm{E}$, against $\mathrm{T}_{2}$. The total heat or internal energy for any temperature can therefore be found by a direct reading.

\section{Use of the Gas Mixture and Nozzle Diagrams.}

(8) In all the types of calculation classified in $\$ 1$, the fundamental operation is, when two of the properties of state are known, to find a third. To use the Diagram, a straight-edge is placed across the scales, intersecting two of them, representing the known properties, at points corresponding to their respective values. The intersection with the third scale of the group then gives the value of the property which it represents. All the other properties may be evaluated, either by repeating the operation of alignment with the other groups of scales, or, in the case of the properties $T$, $\mathrm{H}, \mathrm{E}$, when one of them is known, by a direct reading.

(9) The scales can be read more easily and accurately by the use of a flat strip of transparent material with a fine straight line on the under surface, rather than a straight-edge of a type suitable for drawing. Ruled strips of celluloid, in lengths of $12 \mathrm{in.}$ and 18 in., can be obtained for this purpose, under the name of "Nomograph Rules," or a line may be ruled on a celluloid setsquare of suitable size. Tracing paper and cloth are liable to distortion, but sometimes convenient for temporary use. 
(10) In describing the use of Fig. 1, the operation of $\$ 1$ will be taken in detail, and in the order there adopted.

(a) Calculation of the properties of state when the values of any independent pair of then are known.

(i) From the pressure and specific volume, to find the temperature.-Set the straight-edge at the pressure on scale $\mathrm{P}$ and the specific volume on scale $V$. Read the temperature on scale $T_{1}$. This is the temperature in degrees $F$. absolute; for degrees $F$., subtract 460 .

Similarly, the specific volume can be found for a given pressure and temperature, or the pressure for a given specific volume and temperature, by the use of the same group of scales.

(ii) From the pressure and temperature, to find the entropy.Set the straight-edge at the pressure on scale $P$ and the temperature on scale $T_{2}$. Read the entropy on scale $\phi$.

Similarly, any of the properties pressure, entropy, temperature can be found from given values of the other two by means of this group of scales, $\mathrm{P}, \phi, \mathrm{T}_{\mathrm{g}}$.

(iii) From the specific volume and temperature, to find the entropy.-Set the straight-edge at the specific volume on scale $\mathrm{V}$ and the temperature on scale $T_{3}$. Read the entropy on the scale $\phi$.

Similarly, any of the properties specific volume, entropy, temperature can be found from given values of the other two, by means of the group of scales, $\mathrm{V}, \phi, \mathrm{T}_{3}$.

(iv) From sufficient daita, to find the total heat and internal energy. - If the temperature is known, read total heat on scale $\mathrm{H}$ from temperature on scale $\mathrm{T}_{1}$ and internal energy on scale $\mathrm{E}$.

Similarly, the temperature and thenoe the internal energy can be found from the total heat, or the temperature and total heat from the internal energy.

If none of these properties is known, first find the temperature, by one of the operations (i), (ii), or (iii), and then proceed as above.

It will be seen that the use of the Diagram in the other classes uf calculation resolves itself into different applications of the operations described under class (a).

(b) Calculation of heat received or rejected.

(i) Heat received at constant pressure.-The heat received at constant pressure is equal to the increase in total heat. Therefore 
it is necessary to find the total heat in the initial and final states, and subtract the former value from the latter. The result is the heat received, per pound of mixture.

If the known quantities are the initial state and the heat receired, the addition of the latter to the initial total heat will
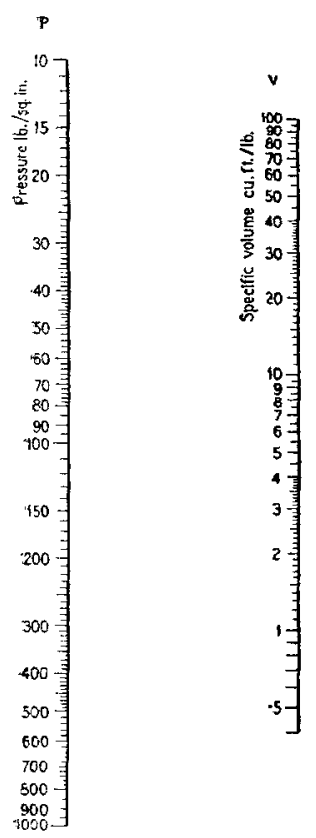

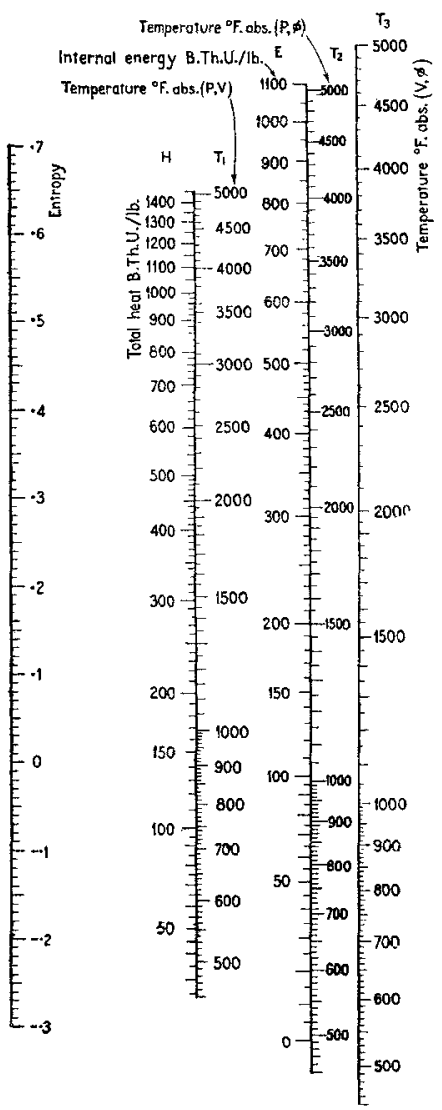

Fig. 1.

give the final total heat, and therefore the final temperature. The final pressure is equal to the initial pressure, so that all the properties of the final state may be found by the operattions already described. In the same way, the properties of any intermediate state may be found, for example, the entropy at 
different temperatures, for the plotting of a constant pressure curve on a temperature-entropy diagram.

In this case, the straight-edge is moved as if pivoted at the pressure scale, over the known constant pressure, and the other
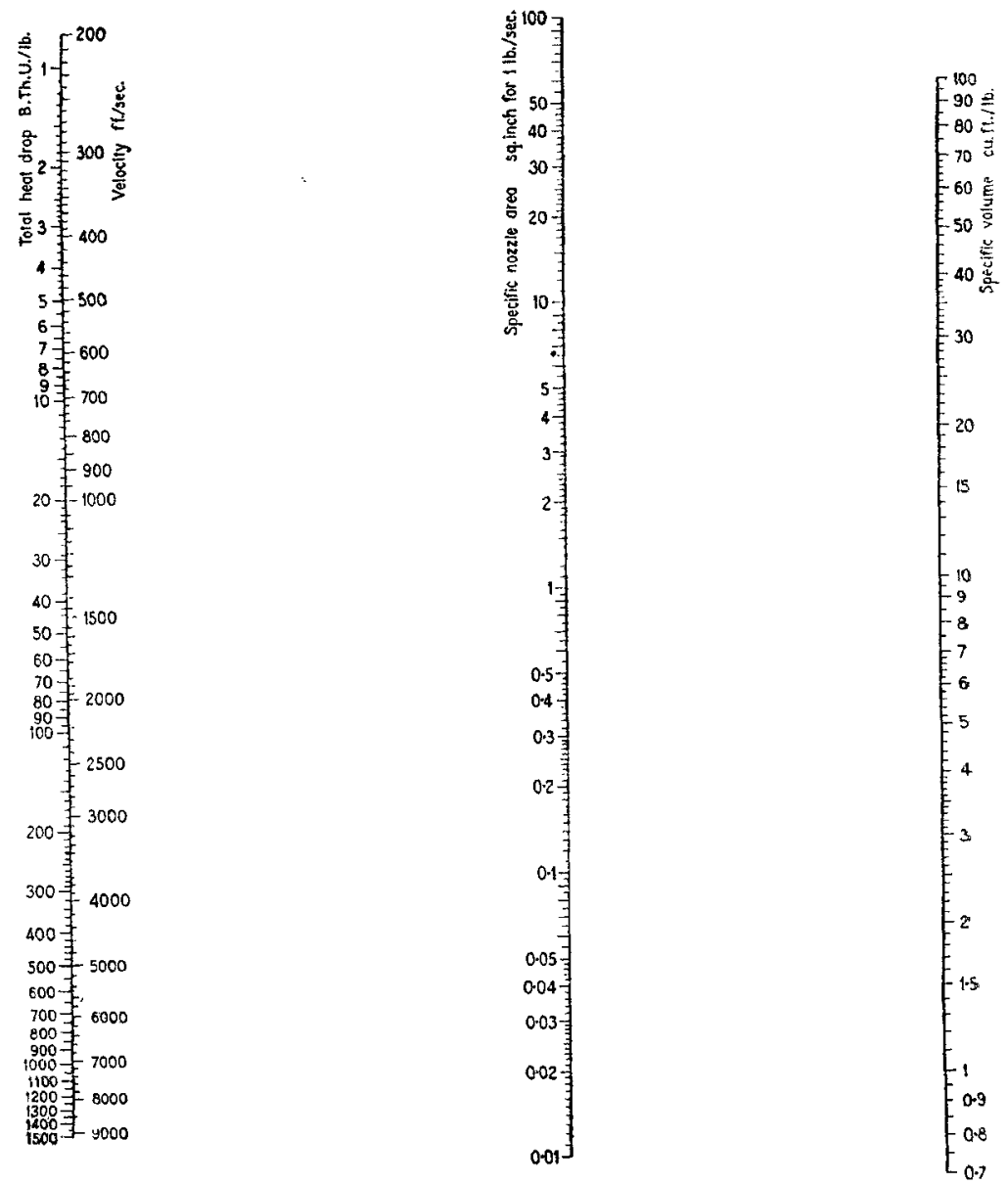

Fra. 2.

properties are read off by aligning with temperature on the appropriate scale. Thus for entropy and temperature, the reading would be on the scale $\phi$ and the setting on the scale $\mathrm{T}_{2}$, or vice versâ. If desired, the straight-edge may be definitely piroted at the pressure by means of a fine needle or pricker. 
The heat rejected at constant pressure is equal to the decrease in total heat, and may be calculated in a similar way.

(ii) The heat received or rejected at constant volume is equal to the increase or decrease in the internal energy. Constantvolume changes can therefore be calculated by similar methods to those adopted in the case of constant pressure, substituting internal energy for total heat, and observing that specific volume, instead of pressure, remains the same.

In this case, then, intermediate readings of temperature and entropy are obtained by pivoting the straight-edge at the constant specific volume on scale $\mathrm{V}$ and reading temperature and entropy on scales $\mathrm{T}_{3}$ and $\phi$ respectively.

(iii) The heat reseived at constant temperature is equal to the increase in entropy multiplied by the temperature.

If the initial and final pressures and the constant temperature are known, the straight-edge is pivoted at the temperature on scale $\mathrm{T}_{2}$, and set at the pressures on seale $\mathrm{P}$, giving the initial and final entropies on scale $\phi$.

Similarly, the calculation may be made from the initial and final volumes, by pivoting on the scale $\mathrm{T}_{3}$, setting on scale $\mathrm{V}$, and reading on scale $\phi$.

The increase in entropy, so obtained, is then multiplied by the temperature. The result is the heat received per pound of mixture.

If the known quantities are the initial state and the heat received, the heat should be divided by the constant temperature, giving the increase in entropy. This should be added to the initial entropy, giving the final entropy, from which, with the known constant temperature, all the other properties of the final state may be obtained, by means of the operations (ii), (iii), and (iv) in class (a).

The heat rejected at constant temperature is equal to the decrease in entropy multiplied by the temperature, and may be calculated in a similar manner.

(c) Calculation of work done in expansion, or received in compression.

(i) In expansion at constant entropy (adiabatic expansion), the work done is equal to the decrease in internal energy, multiplied by the mechanical equivalent of heat, or 778 .

From the known properties of the initial state, the initial GIBSON. 
temperature and entropy are found by the methods of class (a). If the final specific volume is known, pivot at the entropy on scale $\phi$, set at the final specific volume on scale $V$, and read the final temperature on scale $\mathrm{T}_{3}$. From the temperatures, read the internal energies on scale $\mathrm{E}$, and subtract the final from the initial value. The difference, multiplied by 778 , gives the work done in the expansion, in foot-pounds per pound of mixture.

If the final pressure is known, instead of the final volume, the straight-edge is again pivoted at the entropy on scale $\phi$, but set at the final pressure on scale $\mathrm{P}$, and the final temperature is read on scale ' $\mathrm{T}_{2}$, or the final internal energy may be read directly, as the scales $\mathrm{E}$ and $\mathrm{T}_{2}$ are plotted on opposite sides of the same line.

To find the specific volume and pressure at any point on an adiabatic expansion or compression, pivot the straight-edge at the constant entropy on scale $\phi$. If it is desired to find the pressure corresponding to a given specific volume, as in plotting an. adiabatic curve on a pressure-volume diagram, set at the specific volume and read the temperature on scale $\mathbf{T}_{3}$. Then move the straight-edge, still pivoting at the entropy, until the same temperature is indicated on scale $T_{2}$. The required pressure is given nu scale P.

Similarly, the temperature and specific volume may be found from the constant entropy and any given pressure, or the specifio volume and pressure from the constant entropy and a given temperature.

The work received in compression is equal to the work done in the opposite expansion, or to the increase in internal energy multiplied by 778 .

(ii) In expansion at constant temperature (isothermal expansion) the work done is equal to the heat received, multiplied by the mechanical equivalent of heat, as there is no change in the: total heat or internal energy.

The heat received is equal to the increase in entropy multiplied by the constant temperature, and is calculated by the methods of section (iii), class (b). The result is multiplied by 778 , giving the work done, in foot-pounds per pound of mixture.

To find corresponding specific volumes and pressures for an isothermal expansion at known temperature, pivot at the temperature on scale $T_{1}$ and read the specific volume on scale $V$ and the corresponding pressure on scale $\mathrm{P}$.

In an isothermal compression, the work received is equal to the 
heat rejected, multiplied by the mechanical equivalent of heat, and may therefore be found in the same way as the work done in an isothermal expansion.

(iii) In expansion at constant pressure the work done, in footpounds, is equal to the increase in volume, in cu. ft., multiplied by the pressure, in $\mathrm{Ib}$. per sq. $\mathrm{ft}$. To obtain this with the units ased for the Diagram, subtract the initial specific volume from the final specific volume and multiply the result by the pressure and again by 144 . This gives the work done, in foot-pounds per pound of mixture.

To find the mean pressure of any expansion or compression divide the work done, or received, per pound of mixture, by the difference between the final and initial specific volumes, and again by 144 . The result is the mean pressure, in lb. per sq. in.

Similarly, the mean effective pressure of an ideal or other cycle may be obtained by dividing the work, in foot-pounds per pound of mixture by the difference between the maximum and minimum specific volumes of the cycle, and by 144 .

The efficiency of a cycle is found by subtracting the heat rejected from the heat received, and dividing by the heat received, and may therefore be found by operations of class (b).

\section{(d) Transfer of working substance from one vessel to another, and flow through nozzles or orifices.}

In flow through a nozzle or orifice from one vessel to another, in which the pressure is lower, a gas or vapour receives kinetic energy. When the flow is adiabatic, this energy is drawn from the internal and external energies of the substance; if the flow is also frictionless, the kinetic energy at any point in the nozzle is equal to the mechanical equivalent of the drop in the sum of these energies, from the initial state to that point; or, in other words, to the drop in total heat multiplied by the mechanical equivalent of heat.

If $v$ is the velocity in feet per second, the kinetic eniergy per pound will be $v^{2} / 2 g$; therefore, taking $\mathrm{H}^{\prime}$ as the initial total heat, and $\mathrm{H}^{\prime \prime}$ as the total heat at the point considered, we have, in frictionless adiabatic flow through a nozzle of suitable form,

$$
\begin{aligned}
v^{2} & =\left(\mathrm{H}^{\prime}-\mathrm{H}^{\prime \prime}\right) 778 \times 64 \cdot 4 \\
v & =223.8 \sqrt{\mathrm{H}^{\prime}-\mathrm{H}^{\prime \prime} .}
\end{aligned}
$$

If $V^{\prime \prime}$ is the corresponding specific volume, a nozzle to pass one pound per second will have at this point a cross-sectional area of u 02 
$V^{\prime \prime} / v$ square feet, or, substituting for $v$ and multiplying by 144 , $144 \mathrm{~V}^{\prime \prime} / 223 \cdot 8 \sqrt{\mathrm{H}^{\prime}-\overline{\mathrm{H}}^{\prime \prime}} \mathrm{sq}$. in.

A second Diagram, Fig. 2, has been prepared for the calculation of velocity from heat-drop, and of unit nozzle area from velocity (or heat-drop) and specific volume, the expressions above being represented by logarithmic scales on an alignment diagram. It will be understood that this Diagram can be used for problems on the flow of any gas or vapour, if the heat-drop and specifice volume can be found.

As the pressure in the nozzle falls away from the initial pressure, the area required for unit flow decreases until it reaches a minimum at the "throat pressure," and then increases again; when the final pressure is below the throat pressure and it is desired to expand completely before leaving the nozzle, the area of the downstream end of the nozzle is made greater than that of its "throat" or narrowest section.

If $P$ is the initial pressure of the gas mixture, at rest in the high pressure vessel, and $\mathrm{P}_{t}$ the throat pressure, the ratio $\mathrm{P}_{t} / \mathrm{P}^{\prime}$ can be calculated from the properties of the mixture. With specific heats as used in the construction of the gas mixture Diagram, it is given in terms of the initial temperature, over the range plotted on the Diagram, by the approximate formula

$$
\mathbf{P}_{t} / \mathbf{P}^{\prime}=0.5236+0.0000054 \mathrm{~T}
$$

or, with sufficient accuracy for a range of initial temperature from $2000^{\circ}$ to $4000^{\circ} \mathrm{F}$. Abs., it may be taken as 0.54 .

If the final pressure is greater than the throat pressure, the nozzle will be convergent. To find the final area, first obtain the difference between the initial and final total heats, and also the final specific volume, by means of operations (i) in class (c), above. Then, aligning the former on the heat-drop scale and the latter on the specific volume scale of the nozzle Diagram, the area for a flow of one pound per second can be read from the area seale. The final velocity is also given against the heat-drop, on the velocity scale. The area of the ideal nozzle increases to infinity at the upstream end, where the heat-drop and velocity are zero. Actually, the upstream end of a nozzle is rounded off sufficiently to avoid any disturbance to the flow.

If the final pressure is less than the throat pressure, the final area may be found in the same way, but, to obtain complete expansion within the nozzle, it is necessary to provide a throat, making the nozzle divergent. To find the area at the throat, find 
the drop in total heat and the specific volume after expansion from the initial pressure to the throat pressure, and apply the results to the nozzle Diagram as before.

As the throat pressure establishes itself in the neighbourhood of the smallest section of the nozzle, the throat area determines the flow, and the final area the extent of expansion.

In non-adiabatic expansion, heat received must be added to the heat-drop, or heat rejected subtracted from it, to obtain the heat which is converted into kinetic energy.

To apply the ideal nozzle, calculated by the methods given above, to the determination of dimensions for an actual nozzle in design, experimental coefficients must be used. At present, it is necessary to use coefficients based on nozzle experiments with other substances, and principally with steam.

\section{Numerical Examples.}

(11) The following numerical examples have been chosen to demonstrate the principal uses of the Diagrams.

(a) To find the temperature, entropy, total heat, and internal energy of a pound of the gas mixture, occupying a volume of $3.5 \mathrm{cu}$. ft. at a pressure of $375 \mathrm{lb}$. per sq. in. absolute.

Align 375 on scale $P$ with 3.5 on scale V. Read on scale $\mathrm{T}_{1} \quad$ Temperature $=3460^{\circ} \mathrm{F}$. Abs. on scale $\mathrm{H}$ Total heat $=910$ B.Th.U.

Pivot at 375 on scale $P$, and align with 3460 on scale $T_{2}$. Read on scale $\mathrm{E} \quad$ Internal energy $=666 \mathrm{~B}$.Th. $\mathrm{U}$. on scale $\phi \quad$ Entropy $=0.324$

This is the method to be adopted in transposing an experimental pressure-volume (indicator) diagram to other scales, such as temperature-entropy.

For that purpose, first divide the actual volumes, in cubic feet, by the weight of the charge, in pounds, thus expressing them as specific volumes. Then proceed as above. It is, of course, unnecessary to read the properties which are not required.

(b) To find the thermal efficiency and mean effective pressure of the following ideal cycle.

The charge, initially at atmospheric pressure $(14.7 \mathrm{lb}$. per sq. in.) and $160^{\circ} \mathrm{F} .\left(620^{\circ} \mathrm{F}\right.$. Abs.), is compressed adiabatically to 0.065 of its original volume. Heat is received at constant pressure, in the proportion of $600 \mathrm{~B}$.Th.Us. per pound. The charge is 
then expanded adiabatically to the initial volume, and cooled at constant volume to the initial pressure.

The cooling replaces the release to exhaust of the actual cycle, as, in the ideal cycle, the charge is regarded as remaining in the eylinder.

It is convenient to work such problems as for one pound of gas mixture.

\section{Initial State.}

Align 14.7 on scale $P$ with 620 on scale $T_{1}$.

Read on scale $V \quad$ Volume $=16^{\circ} 0 \mathrm{cu}$. ft. per lb.

Pivot at $14 \cdot 7$ on scale $P$ and align with 620 on scale $T_{2}$.

Read on scale $\mathrm{E}$ Internal energy $=23.5 \mathrm{~B}$.Th. Us. per lb.

on scale $\phi \quad$ Entropy $=0.060$

\section{Adiabatic Compression.}

Volume at end of compression $=16^{\circ} 0 \times 0.065=1.04 \mathrm{cu}$. ft. per lb. In adiabatic changes the entropy is constant.

Pirot at 0.060 on seale $\phi$ and align with 1.04 on scale $V$.

Read on scale $\mathrm{T}_{3} \quad$ Temperature $=1630^{\circ} \mathrm{F}$. Abs.

Pivot at 1.04 on scale $\mathrm{V}$ and align with 1630 on scale $\mathrm{T}_{1}$.

Read on scale $H \quad$ Total heat $=342$ B.Th.Us. per Ib.

on scale $P \quad$ Pressure $=595 \mathrm{lb}$. per sq. in.

\section{Reception of Heat at Constant Pressure.}

Heat received at constant pressure $=$ increase in total heat. 600 B.Th.Us. increase the total heat to $942 \mathrm{~B}$.Th.Us. per lb.

Against 942 on scale $\mathrm{H}$, read on scale $\mathrm{T}_{1} \quad$ Temperature $=3550^{\circ} \mathrm{F}$. Abs.

Align 3550 on scale $T_{2}$ with 595 on scale $\mathrm{P}$. Read on scale $\phi \quad$ Entropy $=0.300$

\section{Adiabatic Explansion.}

The final volume is $16^{\circ} 0 \mathrm{cu}$. ft. per $\mathrm{lb}$.

Pirot at 0.300 on scale $\phi$, and align with $16^{\circ} 0$ on scale V. Read on scale $\mathrm{T}_{3} \quad$ Temperature $=2020^{\circ} \mathrm{F}$. Abs.

Against 2020 on scale $T_{2}$, read on scale $\mathrm{E} \quad$ Internal energy $=315 \mathrm{~B}$.Th.Us. per $\mathrm{Ib}$.

Rejection of Heat at Constant Volume.

Heat rejected at constant volume $=$ decrease in internal energy. 
This operation completes the cycle. The final internal energy is equal to the internal energy of the initial state, or to 23.5 B.Th. Us. per lb. and heat rejected $=315-2355=2915 \mathrm{~B}$.Th. Us. per lb.

No heat was received or rejected during compression or expansion, so that the heat converted to work

$$
=600-291.5=308.5 \text { B.Th.Us. per lb. }
$$

The thermal efficiency is therefore $308.5 / 600$, or 51.5 per cent. The "swept volume" or difference between maximum and minimum volumes, is $16^{\circ} 0-1 \cdot 04=14^{\cdot} 96 \mathrm{cu}$. ft. per $1 \mathrm{~b}$.

The mean effective pressure of the cycle is therefore

$$
(308.5 \times 778) /(14.96 \times 144)=111.5 \mathrm{lb} \text {. per sq. in }
$$

In this example, the working has been given at length in order to show the sequence of operations and the reason for each. In making such calculations for ordinary purposes, it is more convenient to put each result, as read, in a short tablle of properties: of state, as under:-

\begin{tabular}{cccccccc} 
State & $\mathrm{P}$ & $\mathrm{V}$ & \multicolumn{1}{c}{$\mathrm{T}$} & $\phi$ & $\mathrm{H}$ & $\mathrm{E}$ & Heat transfer \\
1 & $14 \cdot 7$ & $16 \cdot 0$ & 620 & $0 \cdot 060$ & & $23 \cdot 5$ & \\
2 & 595 & $1 \cdot 04$ & 1630 & $0 \cdot 060$ & 342 & & Rec'd. 600 \\
3 & 595 & & 3550 & $0 \cdot 300$ & 942 & & \\
4 & & $16 \cdot 0$ & 2020 & $0 \cdot 300$ & & 315 & Rej'd. $291 \cdot 5$ \\
1 & $14 \cdot 7$ & $16 \cdot 0$ & 620 & $0 \cdot 060$ & & $23 \cdot 5$ & Heat used 308.5
\end{tabular}

Swept volume $14 \cdot 96$

Heat used $308 \cdot 5$

Cycle thermal efficiency $=308.5 / 600=0.515=51.5$ per cent.

Cycle mean effective pressure $=\frac{308.5 \times 778}{14 \cdot 96 \times 144}=111.5 \mathrm{lb}$. per sq. in.

To estimate the indicated thermal efficiency and mean effective pressure which may be expected in an actual engine, it is necessary to multiply the ideal figures by a "diagram factor," based on experiments.

Alexander, in the paper already mentioned,* gives diagram factors for gas and Diesel engines. The value obtained from the results of tests on a four-cycle gas engine was approximately 0.80 . Two four-cycle Diesel engines, one of small size and the other a large marine engine, showed diagram factors of 0.86 . Iu the case of the larger Diesel engine, the actual indicated thermal efficiency was not obtained from the fuel consumption, but was

* See last footnote on p. 662 . 
deduced from the actual temperature-entropy diagram by a method which gave excellent comparative results when applied to the tests of the other engines.

For a two-cycle oil engine of the mixed combustion type, which corresponds to an ideal cycle in which part of the heat is received at constant volume and the remainder at constant pressure, diagram factors of about 0.80 have been found, the whole stroke being regarded as effective in expansion and compression when constructing the ideal cycle.

These values are substantially in agreement, and, subject to modification by further experiment, may be of use in the application of ideal cycles. It must be understood that these factors are for a condition of good combustion. No allowance is made for unburnt fuel in constructing the ideal cycle; the diagram factor, defined as the ratio of actual indicated thermal efficiency to thermal efficiency of ideal cycle, is therefore reduced if the combustion is incomplete, as when running at abnormal loads.

(c) To find the dimensions of a nozzle to expand 10lb. of gas mixture per second adiabatically to a final pressure of $14.7 \mathrm{lb}$. per sq. in., the initial pressure being $300 \mathrm{lb}$. per sq. in. and the initial temperature $2500^{\circ} \mathrm{F}$. Abs. Also, to find the final velocity in such a nozzle.

\section{Using the Gas Mixture Diagraw.}

For the Initial State.

Against 2500 on scale $\mathrm{T}_{1}$, read on scale $\mathrm{H} \quad$ Total heat $=605$ B.Th.Us. per $\mathrm{lb}$.

Set at 300 on scale $P$ and align with 2500 on scale $T_{2}$. Read on scale $\phi \quad$ Entropy $=0.235$.

State at throat.

Taking the throat pressure as 0.54 of the initial pressure, we have Pressure $=300 \times 0.54=162 \mathrm{lb}$. per sq. in.

Pivot at 0.235 on scale $\phi$ and align with 162 on scale $P$. Read on scale $\mathrm{T}_{2} \quad$ Temperature $=2160^{\circ} \mathrm{F}$. Abs.

Against 2160 on scale $T_{1}$,

read on scale $H$ Thtal heat $=503$ B.Th.Us. per lb.

Pivot at 0.235 on scale $\phi$ and align with 2160 on seale $T_{3}$. Read on scale $V \quad$ Specific volume $=5.15 \mathrm{cu}$. ft. per lb.

Final state.

Pivot at 0.235 on seale $\phi$ and align with 14.7 on scale $P$. Read on scale $\mathrm{T}_{2} \quad$ Temperature $=1200^{\circ} \mathrm{F}$. Abs. 
Against 1200 on scale $T_{1}$,

read on scale $H \quad$ Total heat $=221$ B.Th.Us. per $1 b$.

Pivot at 0.235 on scale $\phi$ and align with 1200 on scale $\mathbf{T}_{3}$. Read on scale $\mathrm{V} \quad$ Specific volume $=31 \mathrm{cu}$. ft. per $\mathbf{l b}$.

We have, from these results, At throat, Heat drop $=605-503=102$ B.Th.Us. per lb. Specific volume $=5 \cdot 15 \mathrm{cu}$. ft. per lb.

Finally, Heat drop $=605-221=384$ B.Th. Us. per lb: Specific volume $=31 \mathrm{cu}$. ft. per lb.

\section{Using the Nozzle Diagram.}

Set at 102 on the heat-drop scale and align with 5.15 on the specific volume scale. Read on the area scale,

Area at throat for $1 \mathrm{lb}$. per sec. $=0.328 \mathrm{sq}$. in.

Set at 384 on the heat-drop scale and align with 31 on the specific volume scale. Read on the area scale,

Final area for $1^{\prime} \mathrm{lb}$. per sec. $=1.018$ sq. in.

Against 384 on the heat-drop scale, read on the velocity scale, Final velocity $=4385 \mathrm{ft}$. per sec.

Multiplying the areas by 10 for a flow of $10 \mathrm{lb}$. per sec., we have the dimensions of the ideal non-conducting and frictionless nozzle for the required expansion,

Area at throat $=3 \cdot 28 \mathrm{sq}$. in.

Final area $=10.18$ sq. in.

and the resulting final velocity,

$4385 \mathrm{ft}$. per sec.

The area and velocity for any intermediate state can be found in the same way.

Experimental factors must be used to reduce these ideal values to correspond with the conditions of an actual nozzle. In the absence of information on the efficiency of nozzles used for the expansion of gas mixture, it is necessary to use the results derived from experiments with steam, taking those which approach most nearly to the conditions of pressure and temperature. It is evident that this method must be used with caution; in the event of gas mixture nozzles for high pressures becoming practically important, experiments under working conditions will be required. 